\title{
Infective Endocarditis Secondary to Granulicatella adiacens
}

\author{
Ahish Chitneni, DO - Annina Giannuzzi, BS² - Kaushik Manthani, DO' - Sandeep A. Gandhi, MD ${ }^{1,2}$
}

A 73-year-old man presented to our emergency department with constipation. He reported that he was unable to have a bowel movement for 5 days prior to presentation, despite the use of home enemas, suppositories, and prune juice. His constipation was associated with chills, decreased oral intake, and decreased urine output.

\section{History}

The patient had a history of hypertension, coronary artery disease, and rheumatoid arthritis and was taking disease-modifying antirheumatic drug therapy. He denied chest pain, fevers, shortness of breath, nausea, vomiting, or joint pain. He denied recent tick bites or international travel. He reported having never received a colonoscopy.

\section{Physical examination}

The patient had poor dentition. His abdomen was soft with normal bowel sounds, and no murmur was appreciated on chest examination. On initial presentation, the patient was afebrile and had tachycardia with a heart rate of 101 beats/min.

\section{Diagnostic testing \\ Initial abdominal radiography scans} showed diffuse bowel ileus vs distal large bowel obstruction. A computed tomography scan of the abdomen and pelvis showed a mildly distended, fluid-filled stomach and small bowel, suggestive of enterocolitis (Figure).

Laboratory study findings were significant for an elevated white blood cell count of $14.67 \times 109 / L$. Initial blood cultures resulted in growth of gram-positive cocci in pairs and chains, but no growth was seen in the urine culture. The patient was initially started on ceftriaxone and vancomycin. Three days after hospital admission, blood cultures grew Granulicatella adiacens, and the patient underwent an echocardiography scan, results of which revealed bacterial endocarditis on the aortic valve.

The patient was educated on the need for outpatient colonoscopy and dental follow-up and was discharged on 4 weeks

\section{AFFILIATIONS:}

${ }^{1}$ Peconic Bay Medical Center, Northwell Health, Riverhead, New York

${ }^{2} \mathrm{New}$ York Institute of Technology College of Osteopathic Medicine, Old Westbury, New York

\section{CITATION:}

Chitneni A, Giannuzzi A, Manthani K, Gandhi SA. Infective endocardititis secondary to Granulicatella adiacens. Consultant. Published online February 23, 2022. doi:10.25270/con.2022.02.00007

Received September 8, 2021. Accepted November 1, 2021.

\section{DISCLOSURES:}

The authors report no relevant financial relationships.

\section{CORRESPONDENCE:}

Sandeep A. Gandhi, MD, 200 Hawkins Avenue, \#1362, Ronkonkoma, NY 11779 (sanganmd@gmail.com)

of intravenous ceftriaxone outpatient parenteral antibiotic therapy.

\section{Discussion}

Infective endocarditis is an infection of the endothelium of the heart resulting from bacteria in the bloodstream. A major risk factor for infective endocarditis is cardiac valvular disease, which enables bacteria in the blood to attach to the damaged heart valves. ${ }^{1}$ Patients with infective endocarditis typically present with fever, fatigue, muscle aches, and a heart murmur on physical examination. Over the last few years, the epidemiology of infective endocarditis has shifted, with Staphylococcus aureus becoming the most prevalent cause in about $26.6 \%$ of cases, Viridans streptococci in $18.7 \%$ of cases, other streptococci strains in $17.5 \%$ of cases, and enterococci strains in $10.5 \%$ of cases. ${ }^{2}$ Infective endocarditis can be associated with bacterial species normally found in the oral flora. Since $G$ adiacens can be found in the oral cavity, it is another potential causative organism in infective endocarditis. ${ }^{1}$

Nutritionally variant streptococci, named for their growth in satellite colonies surrounding other organisms and their unique nutritional requirements, were first discovered in 1961 by Frenkel and Hirsch. ${ }^{3}$ In 1995, Kawamura and colleagues discovered that nutritionally variant streptococci were phylogenetically distinct from other streptococci species using $16 \mathrm{~S}$ rRNA sequencing and reclassified the genus as abiotrophia. ${ }^{4}$ Upon further study, Collins and Lawson found the Abiotrophia genus was not monophyletic and reclassified certain abiotrophia strains, including Abiotrophia adiacens, 


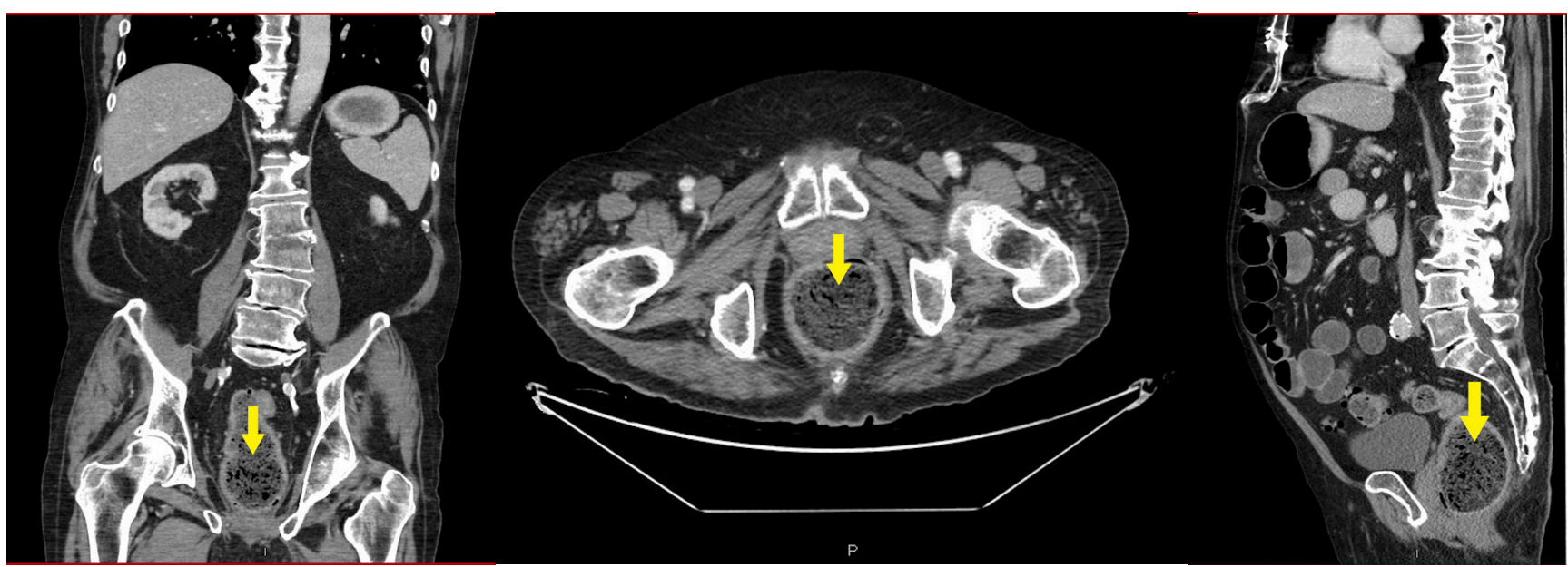

Figure. A computed tomography scan of the abaomen ana peIvis snowea a mılaly aıstenaed tIuıd-TIllea stomacn and small bowel, suggestive of enterocolitis.

into the new genus granulicatella. ${ }^{5}$

$G$ adiacens is a nutritionally variant streptococcus. Growth of the organisms require sulfhydryl compounds such as cysteine and vitamin $B_{6}{ }^{6}$ Typically, $G$ adiacens is part of the normal oral and gastrointestinal flora., ${ }^{7,8}$ One of the complications of infection includes infective endocarditis, for which nutritionally variant streptococci organisms account for less than $5 \%$ of cases and $G$ adiacens account for even fewer cases. ${ }^{9}$ In many cases, the organism may even result in negative blood cultures or delayed identification during the culture process, which may lead to untreated infections. In our case, blood cultures grew $\mathrm{G}$ adiacens within 4 days of hospital admission, which aided in early management; however, that is not always common based on our literature review.

Typically, treatment of $G$ adiacens includes administration of penicillin or ceftriaxone.10 Susceptibility profile studies conducted on $G$ adiacens showed that $34 \%$ of isolates were susceptible to penicillin and $22 \%$ of isolates were susceptible to ceftriaxone.11 In our case, the patient was managed with 4 weeks of ceftriaxone. In addition, research has shown that $G$ adiacens often develop resistance to beta lactam and macrolide antibiotics, which points to further evidence that cephalosporin is ideal for patients with $G$ adiacens infections. ${ }^{12}$
Infective endocarditis is an infection caused by bacteria that enter the bloodstream and affect the heart. Although infective endocarditis is treatable in many cases, some studies have shown that $41 \%$ of cases resulted in failure of treatment of the organism.13 Overall, given the rarity of the organism, potential unique dental findings and gastrointestinal presenting symptoms, the potential for failure of treatment due to resistance, and the potential for $G$ adiacens can cause infective endocarditis. The organism is an important consideration when evaluating patients with similar symptoms. In addition, although many cases in the literature show $G$ adiacens as the cause of endocarditis, no cases in our literature search report a primary gastrointestinal issue as the presenting symptom leading to aortic valve endocarditis.

As mentioned, an echocardiography scan is the primary method to evaluate whether bacteremia has progressed to cause infective endocarditis. Per guidelines published by the European Society of Cardiology (ESC) $)^{10}$ and American College of Cardiology/American Heart Association ${ }^{14}$, a transthoracic echocardiography (TTE) scan is recommended for patients suspected to have infective endocarditis to evaluate for valvular morphology and ventricular function. Suspicion of infective endocarditis is typically based on the Duke criteria (Table) or clin- ical suspicion of organisms that have the potential to cause infective endocarditis. ${ }^{15}$ The Duke criteria consist of major and minor criteria. Major criteria include blood cultures positive for endocarditis (eg, organisms typically consistent with infective endocarditis and evidence of endocardial involvement). Minor criteria include a predisposing heart condition or injection drug use, fever, vascular phenomena (ie, emboli, pulmonary infarcts, intracranial hemorrhage, Janeway lesions), immunologic phenomena (ie, rheumatoid factor, Roth spots, Osler nodes, glomerulonephritis), and microbiological evidence (ie, positive blood culture that does not meet major criteria). Typically after antibiotic treatment, a repeat TTE is conducted to evaluate for resolution of the valvular vegetation and infection. According to the ESC guidelines, a baseline TTE should be performed after the patient completes antibiotic therapy during the first year of follow-up to monitor the development of secondary heart failure. In our case, the patient met the Duke criteria for major criteria in addition to meeting several minor criteria.

We conducted a literature review of cases of endocarditis caused by $G$ adiacens. One case featured an older man who presented with fever and bilateral tingling and numbness in his lower extremities for 15 days. ${ }^{15}$ His physical examination showed a systolic murmur, 
Table. Duke Criteria for Diagnosing Infective Endocarditis ${ }^{2,15}$

\section{MAJOR CRITERIA}

\section{Blood Cultures:}

Typical microorganisms consistent with infective endocarditis from 2 separate blood cultures:

- $\quad$ Viridans streptococci, Streptococcus bovis, HACEK group, Staphylococcus aureus, or community-acquired enterococci with absence of a primary focus

OR

\section{MINOR CRITERIA}

- $\quad$ Predisposing heart condition or intravenous drug use

- Fever of $>38^{\circ} \mathrm{C}$

- Vascular phenomena (including those detected by imaging alone): Arterial emboli, splenic infarction, mycotic aneurysms, intracranial hemorrhage, and Janeway lesions

- Immunological phenomena: Glomerulonephritis, Osler nodes, Roth spots, and rheumatoid factor

- Microbiological evidence: Positive blood cultures not meeting major criteria or serological evidence of infection with organism consistent with infective endocarditis

- $\quad \geq 2$ positive blood cultures drawn > 12 hours apart, or

- All of 3 or a majority of $\geq 4$ separate blood cultures (first and last samples $\geq 1$ hour apart), or

- $\quad$ Single positive blood culture for Coxiella burnetii or phase IgG antibody titer, > $1: 800$

Imaging:

Echocardiography shows:

- Vegetation

- Abscess, pseudoaneurysm, or intracardiac fistula

- Valvular perforation or aneurysm

- New partial dehiscence of prosthetic valve

- Abnormal activity around the site of a prosthetic valve detected by PET/CT scan, assuming $>3$ months after surgery or radiolabeled leukocyte-SPECT/CT

- Definite paravalvular lesions by cardiac CT scan

Abbreviations: HACEK, Haemophilus species, Aggregatibacter species, Cardiobacterium hominis, Eikenella corrodens, and Kingella species; IgG, immunoglobulin G; PET, positron emission tomography; CT, computed tomography; SPECT, single-photon emission computerized tomography.

an echocardiogram showed mitral valve vegetation, and blood cultures grew $G$ adiacens. In this case, the patient had developed severe right lower limb pain on the 6th day of admission. An ultrasonography scan was conducted, results of which showed a thrombus in the right common femoral artery that was thought to be an embolic phenomenon. This case highlights the importance of potential complications from valve vegetations, such as the risk for embolization, and further points to the importance of immediate treatment with antibiotics and thrombosis prophylaxis and follow-up with echocardiography scanning to ensure proper clearance of vegetations.

Another case featured a middle-aged man who presented with fever and mental status changes. ${ }^{16}$ In this case, the patient had blood cultures positive for $G$ adiacens. A computed tomography scan showed left internal jugular vein thrombosis, pulmonary embolism, and abscesses in the gluteal muscles. In this case, the source of the infection was suspected to be $G$ adiacens in the oral flora, which had hematagenously spread to the internal jugular vein with septic metastasis to the lung and the gluteal muscles. Given this patient's unique symptoms and spread of disease, this case further demonstrates the potential complications of $G$ adiacens infection.

Another paper featured 2 cases of the organism resulting in subcutaneous abscesses in the elbow and suprapatellar abscess. ${ }^{17}$ Similar to the previous case, the hematogenous spread of infection from dental or gastrointestinal sources to various regions of the body is important. Although many cases in the literature showed that $G$ adiacens caused infective endocarditis, abscesses, and bacteremia, the gastrointestinal tract as the source of infection is very rare. In our case, our patient presented with constipation, an elevated white blood cell count, and computed tomography imaging showing enterocolitis and stercoralis colitis with perirectal edema and rectal wall thickening. $G$ adiacens should be considered in the differential as a potential cause of infection in similar cases.

\section{References}

1. Ohara-Nemoto $Y$, Kishi K, Satho M, et al. Infective endocarditis caused by Granulicatella elegans originating in the oral cavity. 
J Clin Microbiol. 2005;43(3):1405-1407. doi:10.1128/JCM.43.3.1405-1407.2005

2. Rajani R, Klein JL. Infective endocarditis: A contemporary update. Clin Med (Lond). 2020;20(1):31-35. https://doi.org/10.7861/ clinmed.cme.20.1.1

3. Frenkel A, Hirsch W. Spontaneous development of $L$ forms of streptococci requiring secretions of other bacteria or sulphydryl compounds for normal growth. Nature. 1961;191:728-730. doi:10.1038/191728a0

4. Kawamura Y, Hou XG, Sultana F, Liu S, Yamamoto H, Ezaki T. Transfer of Streptococcus adjacens and Streptococcus defectivus to Abiotrophia gen. nov. as Abiotrophia adiacens comb. nov. and Abiotrophia defectiva comb. nov., respectively. Int J Syst Bacteriol. 1995;45(4):798-803. doi:10.1099/0020771345-4-798

5. Collins MD, Lawson PA. The genus Abiotrophia (Kawamura et al.) is not monophyletic: proposal of Granulicatella gen. nov., Granulicatella adiacens comb. nov., Granulicatella elegans comb. nov. and Granulicatella balaenopterae comb. nov. Int J Syst Evol Microbiol. 2000;50 Pt 1:365-369. doi:10.1099/00207713-50-1-365

6. Bouvet A, Grimont F, Grimont PAD. Streptococcus defectivus sp. nov. and Streptococcus adjacens sp. nov., nutritionally variant streptococci from human clinical speci- mens. Int J Syst Evol Microbiol. 1989;39(3). https://doi.org/10.1099/00207713-39-3-290

7. Lin $\mathrm{CH}$, Hsu RB. Infective endocarditis caused by nutritionally variant streptococci. Am J Med Sci. 2007;334(4):235-239. doi:10.1097/MAJ.0b013e3180a6eeab

8. Aas JA, Paster BJ, Stokes LN, Olsen I, Dewhirst FE. Defining the normal bacterial flora of the oral cavity. J Clin Microbiol. 2005;43(11):5721-5732. doi:10.1128/ JCM.43.11.5721-5732.2005

9. Lin $\mathrm{CH}, \mathrm{Hsu} \mathrm{RB}$. Infective endocarditis caused by nutritionally variant streptococci. Am J Med Sci. 2007;334(4):235-239. doi:10.1097/MAJ.0b013e3180a6eeab

10. Habib G, Lancellotti $P$, Antunes MJ, et al. 2015 ESC Guidelines for the management of infective endocarditis: The Task Force for the Management of Infective Endocarditis of the European Society of Cardiology (ESC). Endorsed by: European Association for Cardio-Thoracic Surgery (EACTS), the European Association of Nuclear Medicine (EANM). Eur Heart J. 2015;36(44):3075-3128. doi:10.1093/eurheartj/ehv319

11. Mushtaq A, Greenwood-Quaintance KE, Cole NC, et al. Differential antimicrobial susceptibilities of Granulicatella adiacens and Abiotrophia defectiva. Antimicrob Agents Chemother. 2016;60(8):5036-5039. doi:10.1128/AAC.00485-16
12. Zheng X, Freeman AF, Villafranca J, et al. Antimicrobial susceptibilities of invasive pediatric Abiotrophia and Granulicatella isolates. J Clin Microbiol. 2004;42(9):43234326. doi:10.1128/JCM.42.9.4323-4326.2004

13. Stein DS, Nelson KE. Endocarditis due to nutritionally deficient streptococci: therapeutic dilemma. Rev Infect Dis. 1987;9(5):908-916. doi:10.1093/clinids $/ 9.5 .908$

14. Nishimura RA, Otto $C M$, Bonow RO, et al. 2014 AHA/ACC guideline for the management of patients with valvular heart disease: a report of the American College of Cardiology/American Heart Association Task Force on Practice Guidelines. J Am Coll Cardiol. 2014;63(22):e57-e185. doi:10.1016/j. jacc.2014.02.536

15. Li JS, Sexton DJ, Mick N, et al. Proposed modifications to the Duke criteria for the diagnosis of infective endocarditis. Clin Infect Dis. 2000;30(4):633-638. doi:10.1086/313753

16. Kawai $H$, Shiojiri T. Internal jugular vein thrombosis associated with Granulicatella adiacens. BMJ Case Rep. 2021;14(1):e238404. doi:10.1136/bcr-2020238404

17. Gupta S, Garg M, Misra S, Singhal S. Granulicatella adiacens abscess: Two rare cases and review. J Lab Physicians. 2018;10(1):121123. doi:10.4103/JLP.JLP_58_17 